\title{
Population-Attributable Fractions of Risk Factors for Hepatocellular Carcinoma in the United States
}

\author{
Tania M. Welzel, MD1 ${ }^{1}$, Barry I. Graubard, PhD², Sabah Quraishi, MPH², Stefan Zeuzem, \\ MD ${ }^{1}$, Jessica A. Davila, PhD ${ }^{3,4}$, Hashem B. El-Serag, MD ${ }^{3,4}$, and Katherine A. McGlynn, \\ $\mathrm{PhD}^{2}$ \\ 1Johann Wolfgang Goethe University Hospital, Frankfurt am Main, Germany \\ ${ }^{2}$ Division of Cancer Epidemiology and Genetics, NCI, NIH, DHHS, Bethesda, Maryland, USA \\ ${ }^{3}$ Houston Veterans Affairs Medical Center, Houston, Texas, USA \\ ${ }^{4}$ Baylor College of Medicine, Houston, Texas, USA
}

\begin{abstract}
OBJECTIVES-Risk factors for hepatocellular carcinoma (HCC) include hepatitis B and C viruses (HBV, HCV), excessive alcohol consumption, rare genetic disorders and diabetes/obesity. The population attributable fractions (PAF) of these factors, however, have not been investigated in population-based studies in the United States.
\end{abstract}

METHODS-Persons $\geq 68$ years diagnosed with HCC $(n=6,991)$ between 1994 and 2007 were identified in the SEER-Medicare database. A $5 \%$ random sample $(n=255,702)$ of persons residing in SEER locations were selected for comparison. For each risk factor, odds ratios (ORs), $95 \%$ confidence intervals (95\% CI) and PAFs were calculated.

RESULTS-As anticipated, the risk of HCC was increased in relationship to each factor: HCV (OR 39.89, 95 \% CI: 36.29-43.84), HBV (OR 11.17, $95 \%$ CI: 9.18-13.59), alcohol-related disorders (OR 4.06, 95 \% CI: 3.82-4.32), rare metabolic disorders (OR 3.45, 95 \% CI: 2.97-4.02), and diabetes and/or obesity (OR 2.47, $95 \%$ CI: 2.34-2.61). The PAF of all factors combined was $64.5 \%$ (males $65.6 \%$; females $62.2 \%$ ). The PAF was highest among Asians (70.1\%) and lowest among black persons (52.4\%). Among individual factors, diabetes/obesity had the greatest PAF (36.6 \%), followed by alcohol-related disorders (23.5 \%), HCV (22.4\%), HBV (6.3\%) and rare genetic disorders $(3.2 \%)$. While diabetes/obesity had the greatest PAF among both males (36.4

(C) 2013 by the American College of Gastroenterology

Correspondence: Katherine A. McGlynn, PhD, DCEG, NCI, NIH, EPS-5022, 6120 Executive Boulevard, Rockville, Maryland 20852, USA. mcglynnk@mail.nih.gov.

SUPPLEMENTARY MATERIAL is linked to the online version of the paper at http://www.nature.com/ajg CONFLICT OF INTEREST

Guarantor of the article: Katherine A. McGlynn, $\mathrm{PhD}$

Specific author contributions: Study concept, data acquisition, analysis, interpretation, and manuscript drafting/revision: Tania M. Welzel, Katherine A. McGlynn, Barry I. Graubard, and Sabah Quraishi; interpretation and manuscript drafting/revision: Jessica A. Davila, Stefan Zeuzem, and Hashem B. El-Serag.

Financial Disclosures: Tania M. Welzel-Consultancies for Novartis. Barry I. Graubard, Sabah Quraishi, Hashem El-Serag, Jessica A. Davila, and Katherine A. McGlynn-None. Stefan Zeuzem-Consultancies for Abbott, Achillion, AstraZeneca, BMS, BoehringerIngelheim, Gilead, Idenix, Janssen, Merck, Novartis, Presidio, Roche, Santaris, and Vertex. 
$\%$ ) and females (36.7 \%), alcohol-related disorders had the second greatest PAF among males (27.8\%) and HCV the second greatest among females (28.1\%). Diabetes/obesity had the greatest PAF among whites (38.9\%) and Hispanics (38.1\%), while HCV had the greatest PAF among Asians (35.4\%) and blacks (34.9\%). The second greatest PAF was alcohol-related disorders in whites (25.6 \%), Hispanics (30.1\%) and blacks (and 18.5 \%) and HBV in Asians (28.5\%).

CONCLUSIONS-The dominant risk factors for HCC in the United States among persons $\ 68$ years differ by sex and race/ethnicity. Overall, eliminating diabetes/obesity could reduce the incidence of HCC more than the elimination of any other factor.

\section{INTRODUCTION}

Primary liver cancer, of which hepatocellular carcinoma (HCC) is the most common histological type, is the sixth most frequently occurring cancer in the world, and the second most common cause of cancer mortality (1). In the United States, the incidence of HCC has almost tripled over the past two decades, with 20,000 individuals being newly diagnosed each year (2). Well-established HCC risk factors include alcoholic liver disease, chronic infection with hepatitis $\mathrm{B}$ and/or $\mathrm{C}$ virus ( $\mathrm{HBV}, \mathrm{HCV})$, and some rare autoimmune or genetic conditions such as autoimmune hepatitis, hereditary hemochromatosis, alpha-1 antitrypsin deficiency, Wilson disease and some porphyrias (3). In concert with the worldwide epidemics of obesity and type 2 diabetes mellitus, increasing evidence suggests a significant contribution of these conditions to HCC risk (4-6). Recently, a large US study identified metabolic syndrome, as well as its individual components (i.e., obesity, diabetes), as significant risk factors for $\mathrm{HCC}$ (7).

The distribution of HCC risk factors in any population may vary over time and in different groups. In addition, estimates of relative risk or odds ratios (ORs) for any of the above HCC risk factors are not capable of describing their contribution to tumor incidence, as the latter is also driven by the prevalence of the factor in the general population. Better characterizations of the impact of the risk factors on the incidence of HCC are the population attributable fractions (PAF), which account both for the strength of the association between exposure and outcome, and also for the prevalence of the exposure in the population. Studies have reported PAFs for HCC in Italy (8-10), Europe (11) and worldwide (12), but PAFs for the US have not been previously reported. Therefore, the goal of the current study was to investigate the PAFs for HCC using data from the SEERMedicare linked database.

\section{METHODS}

Data were obtained from the SEER-Medicare database, which is the linkage of cancer registry data from the National Cancer Institute's Surveillance, Epidemiology and End Results (SEER) program with Medicare medical claims data (13). The SEER program began collating data on cancer cases in 1973, in the states of Connecticut, Iowa, New Mexico, Utah, and Hawaii and the metropolitan areas of Detroit and San Francisco-Oakland. In subsequent years, SEER was expanded to include registries in the Atlanta and Seattle-Puget Sound areas (1974-1975), rural counties in Georgia (1978), American Indians in Arizona (1980), New Orleans (1974-1977, rejoined 2001); New Jersey (1979-1989, rejoined 2001), 
Los Angeles County and the San Jose-Monterey area (1992), and Kentucky and the remaining counties in California (2001). SEER also provides assistance to the Alaska Native Tumor Registry. The data quality and completeness are ascertained regularly.

Medicare is the primary health insurer for $\sim 97 \%$ of individuals aged 65 years and older in the United States. Approximately $99 \%$ of Medicare beneficiaries are covered by Medicare part A benefits, which cover inpatient hospitalizations, and $95 \%$ by part B benefits, which cover outpatient visits and supplier services. The linked SEER-Medicare data set contains part A and part B claims data beginning in 1991 for all Medicare-enrolled patients identified by SEER registries . The linkage captures $\sim 93 \%$ of patients in the SEER database aged 65 years and older.

Cases

All persons diagnosed with $\mathrm{HCC}$ (ICD-O topography code $=\mathrm{C} 22$ and morphology codes $=$ 8170-8175) in a SEER registry who met the following criteria were eligible for inclusion as cases: diagnostic confirmation of HCC, age 65 years or older at diagnosis, and enrolled in Medicare between 1991 and 2007. Diagnostic confirmation was defined as having positive histology, cytology, laboratory test/marker study, direct visualization, or positive radiology tests. Individuals with clinical diagnosis only, unknown method of confirmation, or HCC reported exclusively by death certificate or at autopsy were not eligible for inclusion. To include persons with equal exposure to risk factor information, all individuals had to have continuous enrollment in Medicare parts A and B for at least 3 years before HCC diagnosis. This criterion resulted in a minimum age of 68 years for all participants.

Persons enrolled in a health maintenance organization (HMO) were excluded because Medicare HMO plans have not been required to submit individual claims to CMS for specific services. To avoid the inclusion of metastatic liver disease, all persons with prior diagnoses of stomach, colon, lung, pancreatic, breast, prostate or rectal cancers were excluded.

\section{Controls}

A $5 \%$ random sample of Medicare beneficiaries with no prior cancer diagnosis of HCC who resided in the geographic regions of the SEER-13 registries were selected as controls. The same inclusion/exclusion criteria as used for the cases were applied. A pseudo-diagnosis date using a random number generator was assigned to controls. Controls were matched to cases on the year of search for risk factors to minimize possible diagnostic trends.

\section{Risk factors of interest}

Risk factors were identified for a minimum of 3 years prior to $\mathrm{HCC}$ diagnosis or reference data for the comparison group. The ICD-9 codes used to identify the risk factors are shown in Supplementary Table 1 online. As presence of diabetes and obesity are highly correlated, these conditions were combined in the data analyses.

The study protocol was approved by the Office of Human Subjects Research of the National Institutes of Health, Bethesda, MD. 


\section{Statistical analyses}

Age, race/ethnicity (white, black, Hispanic, Asian, other), geographic region (SEER-13 registry region), and state buy-in status were included as covariates. The state buy-in variable indicates whether a third-party pays a beneficiary's Medicare premiums, and was thus used as an indicator of lower socioeconomic status. Demographic features and preexisting medical conditions were compared between cases and controls using $t$-tests for continuous variables and Chi-square or Fisher's exact tests for categorical variables. Logistic regression was used to calculate ORs and $95 \%$ confidence intervals $(95 \% \mathrm{CI})$. Wald $\chi^{2}$ tests determined the significance of variables in the logistic regressions. Adjusted PAFs for a specific risk factor or set of risk factors were computed using a method for estimating PAFs from population-based case-controls studies with odd ratios for the risk factor set of risk factors adjusted for confounders and other risk factors $(14,15) .95 \%$ confidence intervals were computed using s.e. estimates of adjusted PAFs (16).

All tests of statistical significance were two-sided and a $P$ value $<0.05$ was considered statistically significant. Statistical analyses were performed using SAS Version 9.1 (SAS Institute, Cary, NC). In compliance with SEER-Medicare privacy standards, all cells with fewer than 11 individuals in any table are displayed as " $<11$ ".

\section{RESULTS}

As shown in Table 1, 6,991 individuals with $\mathrm{HCC}$ and 255,702 control individuals were included in the analysis. The greatest percentage of persons with HCC were white (71\%), followed by Asian (12\%), black (8\%), and Hispanic persons (4\%). The majority of the cases $(65.7 \%)$ were male; a proportion which did not vary greatly by racial/ethnic group.

Overall, $22.9 \%$ of the cases were positive for $\mathrm{HCV}, 6.9 \%$ were positive for $\mathrm{HBV}, 31.2 \%$ had prior alcohol-related liver disorders, $4.5 \%$ had rare genetic disorders, $61.5 \%$ had prior diagnoses of diabetes and/or obesity (hereafter referred to as diabetes/obesity) and $19.1 \%$ had no prior diagnosis of any of the above conditions (Table 1). In contrast, $0.4 \%$ of the controls were positive for $\mathrm{HCV}, 0.1 \%$ were positive for $\mathrm{HBV}, 7.4 \%$ had prior alcoholrelated liver disorders, $0.8 \%$ had rare genetic disorders, $34.7 \%$ had a prior diagnosis of diabetes/obesity and $60.5 \%$ had no prior diagnosis of any of the above conditions.

The prevalence of almost all conditions varied greatly by racial/ethnic group. For $\mathrm{HCV}$, the highest rate was found among Asians (cases $=37.1 \%$, controls $=1.7 \%$ ) and the lowest rate, among white persons (cases $=18.5 \%$, controls $=0.4 \%$ ). For HBV, the highest rate was again among Asians (cases $=30.3 \%$, controls $=1.5 \%$ ) and the lowest rate among white persons (cases $=2.8 \%$, controls $=0.1 \%$ ). For alcohol-related liver disorders, the highest percentage was seen among Hispanics (cases $=39.3 \%$, controls $=9.0 \%$ ) and the lowest percentage was seen among Asians (cases $=21.9 \%$, controls $=4.5 \%$ ). For rare genetic disorders, the highest percentage of affected persons occurred among white persons (cases $=5.0 \%$, controls $=$ $0.7 \%$ ) and the lowest percentage occurred among black persons (cases $=<2.1 \%$, controls $=$ $0.8 \%$ ). Diabetes was relatively common in all groups, with percentages ranging from $68.9 \%$ of Hispanic cases and $50.7 \%$ of Hispanic controls to $58.7 \%$ of white cases and $31.0 \%$ of white controls. Percentages of persons who were obese varied more widely, with Hispanics 
having the highest percentages (cases $=13.1 \%$, controls $=7.1 \%$ ) and Asians having the lowest percentages (cases $=2.5 \%$, controls $=2.8 \%$ ). When diabetes and obesity were grouped together, the percentage of affected persons ranged from $71.5 \%$ of Hispanic cases and $52.4 \%$ of Hispanic controls to $61.0 \%$ of white cases and $32.8 \%$ of white controls.

Table 2 displays the OR of each risk factor overall and by gender. Among the total population, HCV had the largest OR at $39.9(95 \% \mathrm{CI}=36.3-43.8)$, followed by HBV (OR $=11.17 ; 95 \% \mathrm{CI}=9.18-13.59)$, alcohol-related disorders $(\mathrm{OR}=4.06,95 \% \mathrm{CI}=3.82$ 4.32), rare genetic disorders $(\mathrm{OR}=3.45,95 \% \mathrm{CI}=2.97-4.02)$, and finally diabetes/obesity $(\mathrm{OR}=2.47,95 \% \mathrm{CI}=2.34-2.61)$. ORs were also calculated after eliminating all risk factors that arose in the 12 months preceding the diagnosis of HCC. No appreciable change was noted in this sensitivity analyis (data not shown).

The PAF of each factor is also shown in Table 2. Overall, the greatest PAF was associated with diabetes/obesity (36.6\%), followed by alcohol-related liver disorders (23.5 \%), HCV $(22.4 \%)$, HBV (6.3\%), and rare genetic disorders (3.2\%). A comparison of PAFs in males and females found that the single greatest PAF in both genders was conferred by diabetes/ obesity (male $=36.4 \%$, female $=36.7 \%$ ). Among males, alcohol-related liver disorders had the second greatest PAF (27.8 \%), followed by HCV (19.3\%). Among females, the order of alcohol-related liver disorders and HCV was reversed with HCV having a PAF of $28.1 \%$ and alcohol-related liver disorders having a PAF of $15.4 \%$. HBV had very similar PAFs in both genders $($ male $=6.4 \%$, female $=6.1 \%$ ). In contrast, rare genetic disorders had a substantially larger role among males $(4.3 \%)$ than among females $(0.9 \%)$. All factors together accounted for a larger proportion of HCC in males (65.6\%) than in females $(62.2 \%)$.

An examination of PAFs by racial/ethnic group found noticeable differences among the groups (Table 3). Among white persons, the factor with the greatest PAF (38.9\%) was diabetes/obesity, followed by alcohol-related liver disorders (25.6\%) and HCV (18.1\%). Among black persons, the factor with the greatest PAF was HCV (34.9\%), followed by alcohol-related liver disorders (18.5\%) and diabetes/obesity (11.5\%). Among Hispanics, diabetes/obesity had the greatest PAF $(38.1 \%)$ followed by alcohol-related liver disorders (30.1\%) and HCV (28.2\%), while among Asians, the factors with the greatest PAFs were HCV (35.4\%), diabetes/obesity (28.5\%) and HBV (28.5\%). The cumulative PAF of these factors was highest among Asians (70.1\%), followed by Hispanics $(69.7 \%$ ), white persons $(64.0 \%)$ and finally, black persons $(52.4 \%)$.

A comparison by time period found that the PAFs of all factors increased in the interval between 1994-1999 and 2000-2007 (Table 4). The combined PAFs of all factors increased from $54.3 \%$ in 1994- 1999 to $65.7 \%$ in 2000-2007. The PAFs of HCV and HBV increased most substantially, with the PAF of HCV increasing from $13.3 \%$ to $25.3 \%$ and the PAF of HBV increasing from $3.1 \%$ to $7.2 \%$. The PAFs of the other factors increased more modestly, with the PAF of alcohol-related liver disorders increasing from $21.4 \%$ to $23.0 \%$, rare genetic disorders from $2.6 \%$ to $3.2 \%$ and diabetes/obesity from $30.0 \%$ to $35.6 \%$. Although diabetes can be an independent risk factor for HCC, it can at times, also be the result of another HCC risk factor. Therefore, cumulative PAFs that did not include diabetes 
were also calculated for the two time periods of interest. For the period 1994-1999, the cumulative PAF for all factors except diabetes/obesity was 34.9\% (31.9-37.9\%). In the time period 2000-2007, the cumulative PAF for all risk factors except diabetes/obesity was $46.0 \%$ (44.5-47.5\%) (data not shown).

\section{DISCUSSION}

This is the first population-based case-control study to report PAFs for HCC risk factors in the United States. The estimate of the cumulative PAF for HBV, HCV, alcohol-related liver disease, diabetes/obesity and some rare genetic disorders indicated that these factors account for $64.5 \%$ (males $65.6 \%$; females $62.2 \%$ ) of the HCC cases in this population. By race/ ethnicity, the combined cumulative PAF was highest among Asians (70.1\%) and lowest among black persons (52.4\%).

The calculation of PAFs for the individual factors indicates that diabetes/obesity had the highest PAF for HCC in both the cumulative (36.6\%) and gender-specific analyses (males $36.4 \%$; females $36.7 \%$ ). PAF estimates for these condition(s) also exceed those for any other HCC risk factor in white persons (38.9\%) and Hispanics (38.1\%), and, together with HBV infection, had the second highest PAF among Asians (28.5\%). This finding emphasizes that despite having the lowest relative risk among the factors examined, the high prevalence of diabetes/obesity in the study population translated into a high attributable fraction. The impact of non-alcoholic fatty liver disease (NAFLD) or non-alcoholic fatty steatohepatitis as the hepatic manifestation of diabetes/obesity has been clearly linked to liver disease progression (fibrosis, cirrhosis) and HCC risk in several clinical or molecular studies (17-20). The recent increase in diabetes, obesity and NAFLD and its impact on HCC incidence highlights the need for effective screening and prevention programs, and the development of strategies to halt liver disease progression in persons with NAFLD. In some instances, of course, diabetes may be the result of another HCC risk factor such as HCV or hemochromatosis (21). As the Medicare data do not contain each person's complete medical history, it was not possible for the current study to determine whether diabetes resulted from another HCC risk factor or simply co-occurred with another factor. It was possible, however, to calculate the PAF of all factors except diabetes/obesity and to compare that result with the PAF of all factors including diabetes/obesity. In both time periods, the difference in PAF was between $19 \%$ and $20 \%$, which represents the percentage of HCC accounted for by diabetes/obesity after all other risk factors have been eliminated.

The impact of obesity on HCC risk was previously assessed in a recent report from the European Prospective Investigation into Cancer (EPIC) cohort (11). Adjusted relative risk estimates for obesity in the EPIC Study and obesity/diabetes in our study population were similar (OR 2.13 and 2.47, respectively). Differences in the PAF for obesity (16.1\% in EPIC) likely reflects varying levels of obesity in the US and Europe, as a recent report found that the prevalence of obesity among US adults was $33.1 \%$, but only $17.1 \%$ among European adults (22). The same report also found a notable difference in the prevalence of diabetes in the US (16.4\%) vs. Europe (10.9\%) (22). 
Alcohol-related liver disorders, well established to promote hepatocarcinogenesis (23), had the second greatest PAF (23.5\%) for HCC in the overall population and among males (27.8 $\%)$. Among women, alcohol-related disorders ranked third (15.4\%). Among racial/ethnic groups, alcohol-related disorders had the second greatest PAF among white persons (25.6 $\%)$, Hispanics (30.1\%), and black persons (18.5\%), but had the fourth greatest PAF among Asians. The gender and racial/ethnic distributions of the PAFs are not unexpected in that they mirror the alcohol consumption patterns in the US population, where males are heavier alcohol consumers than females and Asians are the group least likely to consume any alcohol, or to be heavy drinkers (24).

The PAF estimates for alcohol-related liver disorders are comparable to those described in a previous US single center study in which $32 \%$ of the HCCs were attributable to daily alcohol consumption (25), but lower than the PAFs in an Italian study, in which $45 \%$ of the cancer cases were alcohol-related (9). The current results are similar to a recent report from the EPIC study that reported PAFs for alcohol of $33 \%$ among men and $18 \%$ among women (26).

While the ORs of HCC were far higher for HCV or HBV than for other conditions, the same was not true of their attributable fractions. Because chronic HCV and HBV infections are uncommon in the general population, the PAFs of HCV (22.4\%) and HBV (6.3\%) were only third and fourth highest overall. A greater proportion of HCC among women (28.1\%) than men $(19.3 \%)$, however, was attributable to $\mathrm{HCV}$, while the proportions of HCC attributable to HBV were comparable between the genders. The higher PAF of HCV among women is not unanticipated as other risk factors, such as heavy alcohol consumption and rare genetic disorders, were much less common among women than men. As expected, high PAFs for both HCV and HBV infection were found among Asians. The high prevalence of these infections in Asians of ages 68 years and older may be due to immigration from Asian countries where these viruses are more prevalent $(27,28)$. The high PAF for HCV among black persons (34.9\%) is consistent with data from the National Health and Nutrition Examination Survey that found higher prevalences of HCV infection among non-Hispanic black persons (4.1\%) than among non-Hispanic white persons (3.0\%) or among MexicanAmericans (3.4\%) (29).

Not unexpectedly given their low prevalence in the population, the PAF of rare genetic disorders combined was only $3.2 \%$, although there were differences by gender (males 4.3 $\%$; females $0.9 \%$ ) and racial group (white persons $3.9 \%$; Asians $1.9 \%$ ). PAFs for rare genetic disorders could not be assessed in black persons or Hispanics due to the small number of HCC cases with these conditions. Similarly, the PAF of autoimmune hepatitis could not be assessed as there were no HCC cases with a prior diagnosis of autoimmune hepatitis, a finding consistent with the literature (30).

The PAFs of HCV and HBV almost doubled between 1994-1999 and 2000-2007, while the PAFs of all other conditions increased less dramatically. The overall increases in PAFs suggest that better ascertainment of the studied conditions, as well as increased prevalence in the population or changes in the underlying population, could underlie the trends. The increasing PAF of $\mathrm{HCV}$ is almost certainly due to both its increased prevalence in the 
population and better ascertainment as screening for $\mathrm{HCV}$ infection was not available until the early 1990s. The increased prevalence of obesity and diabetes in the underlying population since the mid-1980 s has been well documented (31).

The current study had notable strengths, as well as some limitations. A major strength was the source of the data. The SEER-Medicare database is population based and covers a representative portion of the US population. As a result, the findings of the current study should be generalizable to the US population aged 68 years and older. The SEER program maintains a case ascertainment completeness rate of at least $98 \%$, thus it is unlikely that the HCC cases in the current study are not representative of all HCC cases in this population. In addition, all diagnoses of HCC in the current study were confirmed by pathology, radiology, and/or laboratory staging. The greatest limitation of the study is that it relied on medical billing records for risk factor information. As a result, many of the medical conditions, in particular obesity, were underestimated. Medical conditions, such as HBV and HCV infection, which are determined by clinical tests, are likely to be more underestimated in controls than cases as the serological tests are not routinely run on all persons. Reliance on medical records data rather than questionnaire data also precluded obtaining more detailed information on behavioral risk factors such as the amount of alcohol consumed and tobaccosmoking patterns. For this reason, the assessment of attributable fractions for tobacco smoking was not possible, although liver cancer has now been included by IARC on the list of cancers causally associated with tobacco smoking (32). Finally, combinations of ICD-9 codes used for risk factor identification were not previously validated.

In conclusion, the results of this population-based study indicate that the dominant risk factors for HCC in the United States may differ by sex and race/ethnicity. Overall, however, eliminating diabetes and obesity could have a larger effect on reducing the incidence of HCC than the elimination of any other risk factor.

\section{Supplementary Material}

Refer to Web version on PubMed Central for supplementary material.

\section{Acknowledgments}

We thank Tim McNeel of Information Management Services, Inc for computer programming and data management support to the project.

Financial support: This work was funded by the Intramural Research Program of the National Cancer Institute, National Institutes of Health.

\section{REFERENCES}

1. Ferlay, J.; Shin, HR.; Bray, F., et al. GLOBOCAN 2008 v1.2, Cancer Incidence and Mortality Worldwide. Lyon, France: IARC;

2. Altekruse SF, McGlynn KA, Reichman ME. Hepatocellular carcinoma incidence, mortality, and survival trends in the United States from 1975 to 2005. J Clin Oncol. 2009; 27:1485-1491. [PubMed: 19224838]

3. McGlynn KA, London WT. The global epidemiology of hepatocellular carcinoma: present and future. Clin Liver Dis. 2011; 15:223-243. vii-x. [PubMed: 21689610] 
4. Calle EE, Teras LR, Thun MJ. Obesity and mortality. N Engl J Med. 2005; 353:2197-2199. [PubMed: 16291995]

5. El-Serag HB, Hampel H, Javadi F. The association between diabetes and hepatocellular carcinoma: a systematic review of epidemiologic evidence. Clin Gastroenterol Hepatol. 2006; 4:369-380. [PubMed: 16527702]

6. Wang C, Wang X, Gong G, et al. Increased risk of hepatocellular carcinoma in patients with diabetes mellitus: a systematic review and meta-analysis of cohort studies. Int J Cancer. 2012; 130:1639-1648. [PubMed: 21544812]

7. Welzel TM, Graubard BI, Zeuzem S, et al. Metabolic syndrome increases the risk of primary liver cancer in the United States: a study in the SEER-Medicare database. Hepatology. 2011; 54:463471. [PubMed: 21538440]

8. Braga C, La Vecchia C, Negri E, et al. Attributable risks for hepatocellular carcinoma in northern Italy. Eur J Cancer. 1997; 33:629-634. [PubMed: 9274446]

9. Donato F, Tagger A, Chiesa R, et al. Hepatitis B and C virus infection, alcohol drinking, and hepatocellular carcinoma: a case-control study in Italy. Brescia HCC Study. Hepatology. 1997; 26:579-584. [PubMed: 9303486]

10. Franceschi S, Montella M, Polesel J, et al. Hepatitis viruses, alcohol, and tobacco in the etiology of hepatocellular carcinoma in Italy. Cancer Epidemiol Biomarkers Prev. 2006; 15:683-689. [PubMed: 16614109]

11. Trichopoulos D, Bamia C, Lagiou P, et al. Hepatocellular carcinoma risk factors and disease burden in a European cohort: a nested case-control study. J Natl Cancer Inst. 2011; 103:16861695. [PubMed: 22021666]

12. Bosch FX, Ribes J, Diaz M, et al. Primary liver cancer: worldwide incidence and trends. Gastroenterology. 2004; 127:S5-S16. [PubMed: 15508102]

13. Warren JL, Klabunde CN, Schrag D, et al. Overview of the SEER-Medicare data: content, research applications, and generalizability to the United States elderly population. Med Care. 2002; 40:IV, 3-18.

14. Bruzzi P, Green SB, Byar DP, et al. Estimating the population attributable risk for multiple risk factors using case-control data. Am J Epidemiol. 1985; 122:904-914. [PubMed: 4050778]

15. Miettinen OS. Proportion of disease caused or prevented by a given exposure, trait or intervention. Am J Epidemiol. 1974; 99:325-332. [PubMed: 4825599]

16. Graubard BI, Fears TR. Standard errors for attributable risk for simple and complex sample designs. Biometrics. 2005; 61:847-855. [PubMed: 16135037]

17. Yasui K, Hashimoto E, Komorizono Y, et al. Characteristics of patients with nonalcoholic steatohepatitis who develop hepatocellular carcinoma. Clin Gastroenterol Hepatol. 2011; 9:428443. [PubMed: 21320639]

18. Starley BQ, Calcagno CJ, Harrison SA. Nonalcoholic fatty liver disease and hepatocellular carcinoma: a weighty connection. Hepatology. 2010; 51:1820-1832. [PubMed: 20432259]

19. Hashimoto E, Tokushige K. Hepatocellular carcinoma in non-alcoholic steatohepatitis: growing evidence of an epidemic? Hepatol Res. 2012; 42:1-14. [PubMed: 21917086]

20. Ascha MS, Hanouneh IA, Lopez R, et al. The incidence and risk factors of hepatocellular carcinoma in patients with nonalcoholic steatohepatitis. Hepatology. 2010; 51:1972-1978. [PubMed: 20209604]

21. Moscatiello S, Manini R, Marchesini G. Diabetes and liver disease: an ominous association. Nutr Metab Cardiovasc Dis. 2007; 17:63-70. [PubMed: 17164082]

22. Thorpe KE, Howard DH, Galactionova K. Differences in disease prevalence as a source of the USEuropean health care spending gap. Health Aff. 2007; 26:w678-w686.

23. Morgan TR, Mandayam S, Jamal MM. Alcohol and hepatocellular carcinoma. Gastroenterology. 2004; 127:S87-S96. [PubMed: 15508108]

24. Chartier K, Caetano R. Ethnicity and health disparities in alcohol research. Alcohol Res Health. 2010; 33:152-160.

25. Hassan MM, Hwang LY, Hatten CJ, et al. Risk factors for hepatocellular carcinoma: synergism of alcohol with viral hepatitis and diabetes mellitus. Hepatology. 2002; 36:1206-1213. [PubMed: 12395331] 
26. Schutze M, Boeing H, Pischon T, et al. Alcohol attributable burden of incidence of cancer in eight European countries based on results from prospective cohort study. BMJ. 2011; 342:d1584. [PubMed: 21474525]

27. Tanaka M, Katayama F, Kato H, et al. Hepatitis B and C virus infection and hepatocellular carcinoma in China: a review of epidemiology and control measures. J Epidemiol. 2011; 21:401416. [PubMed: 22041528]

28. Kurbanov F, Tanaka Y, Elkady A, et al. Tracing hepatitis C and Delta viruses to estimate their contribution in HCC rates in Mongolia. J Viral Hepat. 2007; 14:667-674. [PubMed: 17697020]

29. McQuillan GM, Kruszon-Moran D, Kottiri BJ, et al. Racial and ethnic differences in the seroprevalence of 6 infectious diseases in the United States: data from NHANES III, 1988-1994. Am J Public Health. 2004; 94:1952-1858. [PubMed: 15514236]

30. Trivedi PJ, Cullen S. Autoimmune hepatitis, cirrhosis, and hepatocellular carcinoma (HCC) . Dig Dis Sci. 2011; 56:276-278. [PubMed: 21127980]

31. Wang Y, Beydoun MA. The obesity epidemic in the United States-gender, age, socioeconomic, racial/ethnic, and geographic characteristics: a systematic review and meta-regression analysis. Epidemiol Rev. 2007; 29:6-28. [PubMed: 17510091]

32. Tobacco smoke and involuntary smoking. IARC Monogr Eval Carcinog Risks Hum. 2004; 83:11438. [PubMed: 15285078] 


Study Highlights
WHAT IS CURRENT KNOWLEDGE
$\checkmark \quad$ Hepatocellular carcinoma (HCC) incidence is increasing in the United States.
The risk of HCC varies by sex and ethnicity.
$\checkmark \quad$ Risk factors include excess alcohol consumption, hepatitis C virus infection,
hepatitis B virus infection, diabetes and obesity.
$\checkmark \quad$ The contribution of each risk factor varies by sex and ethnicity.
The attributable fraction of diabetes/obesity, overall, is greater than for any
other risk factor.




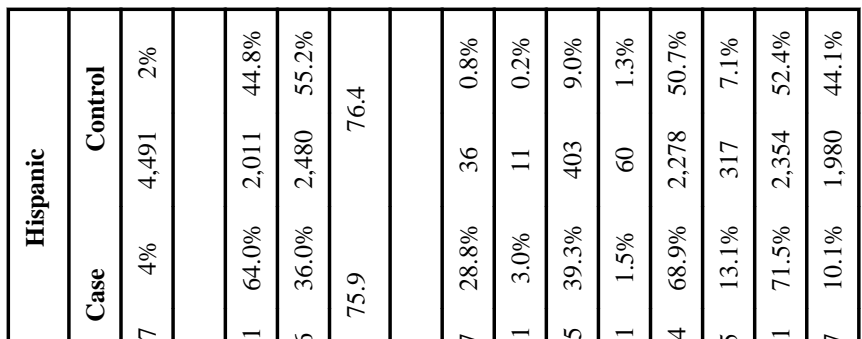

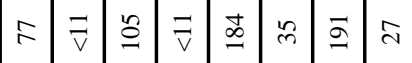
悾

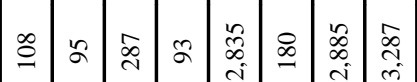

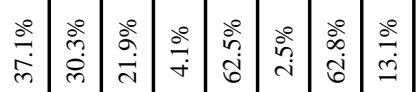
\&

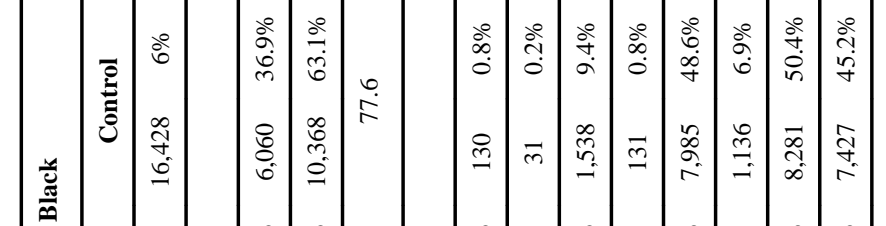

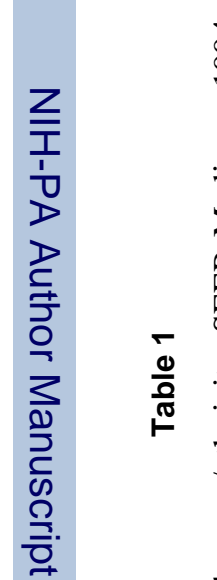

$\frac{2}{2}$ 隹

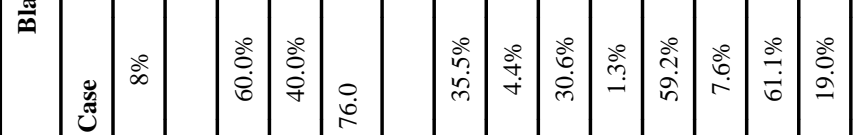

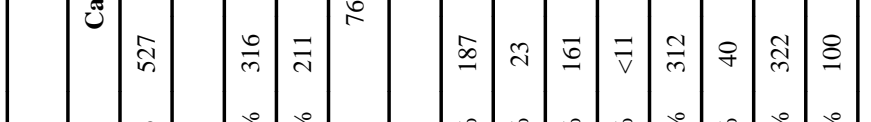

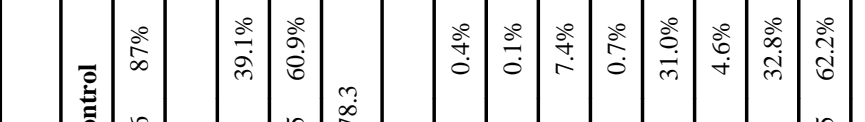

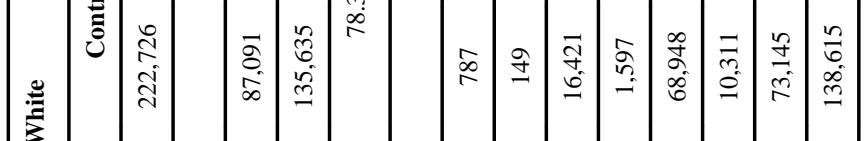

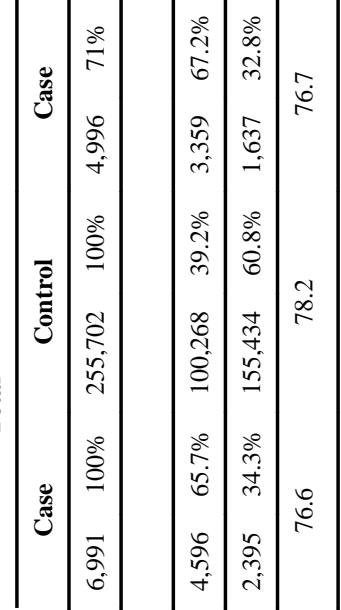




\begin{tabular}{|c|c|c|c|c|c|c|c|c|}
\hline & $\begin{array}{l}\text { Z } \\
\therefore \\
\varkappa\end{array}$ & $\mid \begin{array}{l}0 \\
0 \\
0 \\
0 \\
0 \\
0 \\
0\end{array}$ & 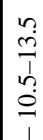 & $\begin{array}{l}a \\
\infty \\
\tilde{f} \\
\stackrel{d}{N}\end{array}$ & $\mid \begin{array}{l}m \\
\stackrel{7}{1} \\
\vdots \\
\grave{d}\end{array}$ & 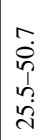 & $\begin{array}{l}\tilde{r} \\
\hat{j} \\
\delta \\
\dot{\gamma}\end{array}$ & 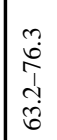 \\
\hline 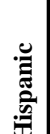 & $\begin{array}{l}0 \\
0 \\
2\end{array}$ & 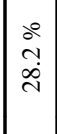 & \begin{tabular}{l}
$\therefore$ \\
\hdashline \\
-1
\end{tabular} & $\frac{\circ}{0}$ & $\begin{array}{l}0 \\
\infty \\
0 \\
0\end{array}$ & $\frac{\circ}{\infty}$ & 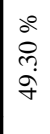 & $\begin{array}{l}\stackrel{2}{0} \\
\hat{a}\end{array}$ \\
\hline
\end{tabular}

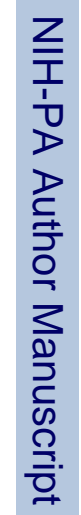

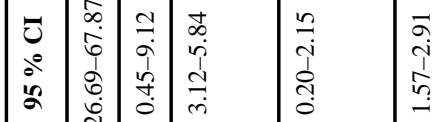

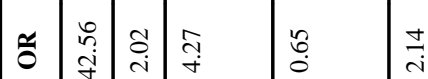

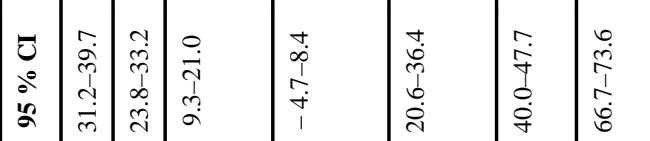
镸

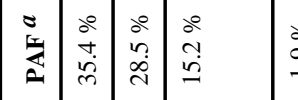

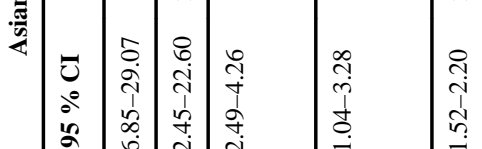

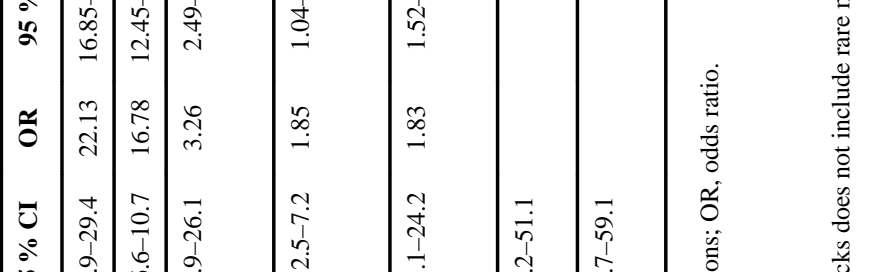

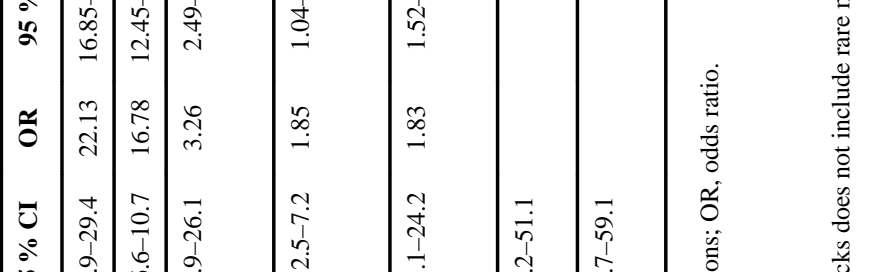
«

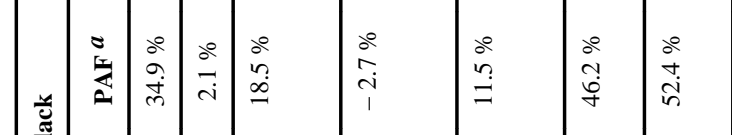
离 要

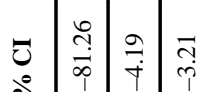

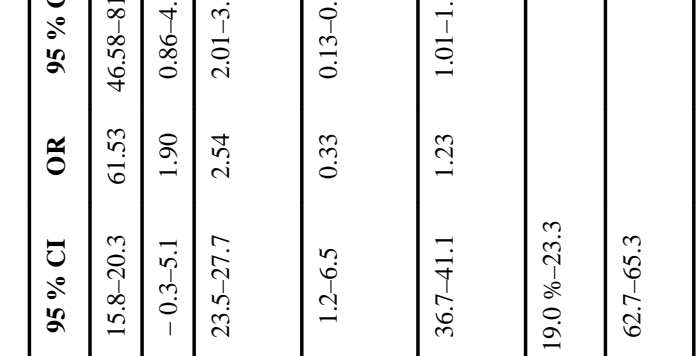

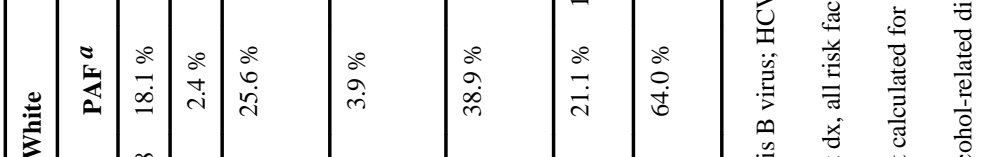

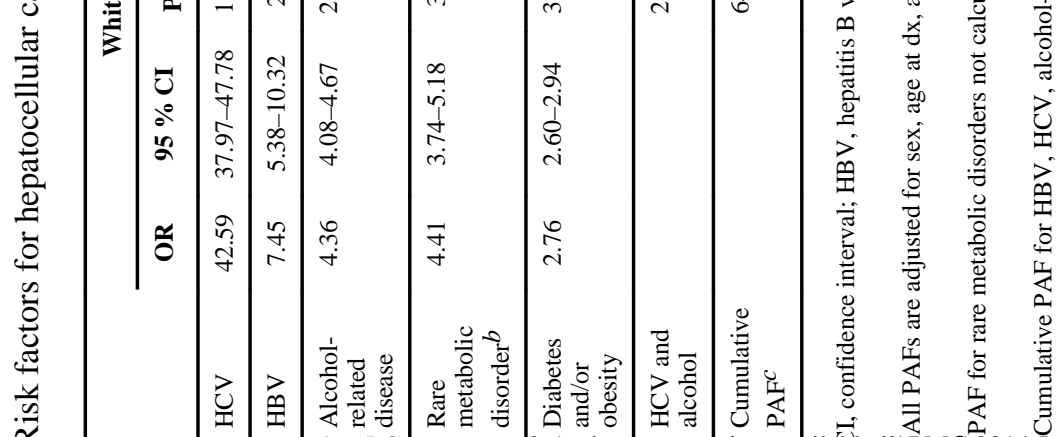




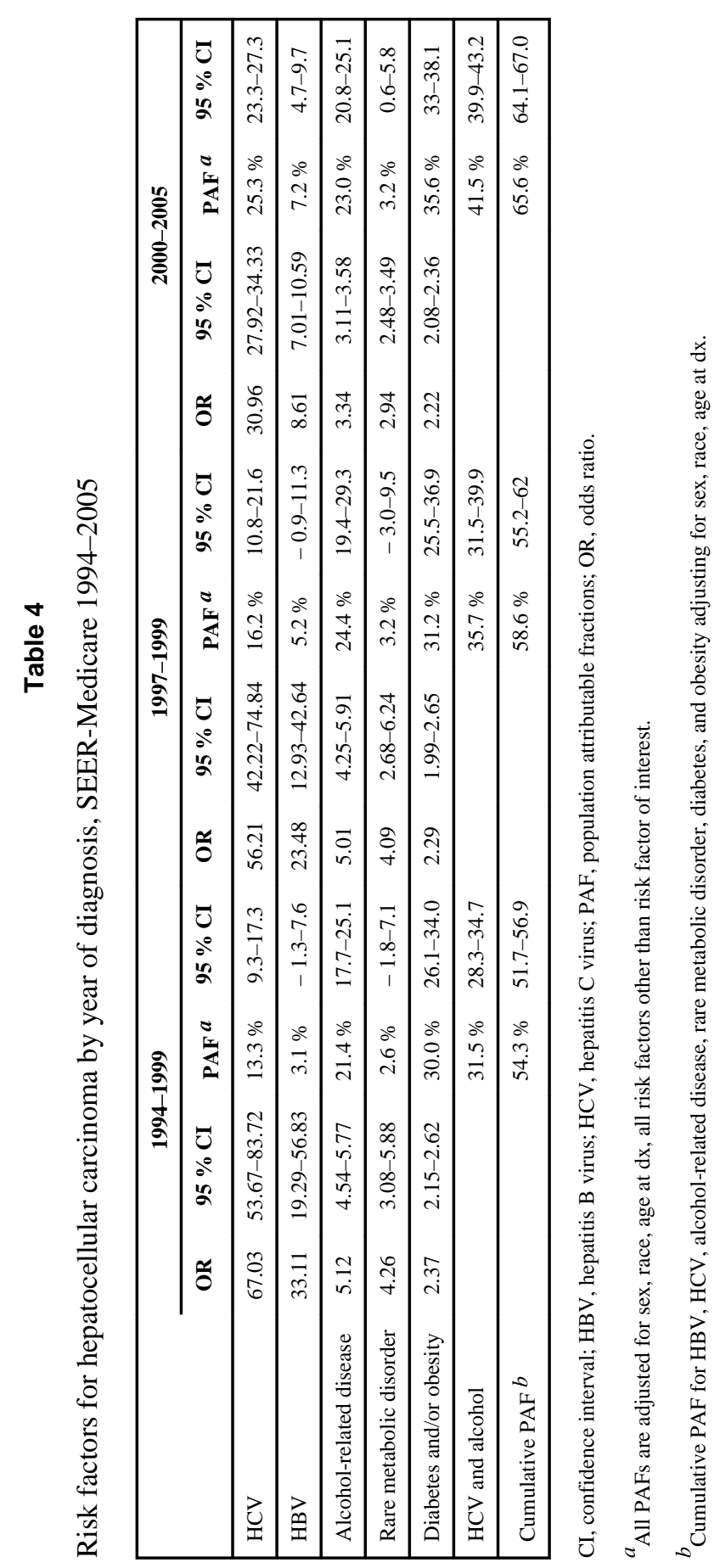

